\title{
Small-scale transcriptomics reveals differences among gonadal stages in Asian seabass (Lates calcarifer)
}

Preethi Ravi ${ }^{1,6 \dagger}$, Junhui Jiang ${ }^{1,2,3 \dagger}$, Woei Chang Liew ${ }^{1,4}$ and László Orbán ${ }^{1,2,5^{*}}$

\begin{abstract}
Background: The Asian seabass (Lates calcarifer) is a protandrous hermaphrodite that typically matures as a male at approximately 2-4 years of age and then changes sex in subsequent years. Although several sexual maturation stages have been described histologically for both testis and ovary, the underlying gene expression profiles remain lacking. The development of a gene expression platform is therefore necessary to improve our understanding of the gonad development of this cultured teleost species.
\end{abstract}

Methods: Thirty Asian seabass gonads were collected from farms in Singapore, examined histologically and staged according to their sex and gonadal maturation status. Partial coding sequences of 24 sex-related genes were cloned using degenerate primers and were sequenced. Additional 13 cDNA sequences were obtained through next-generation sequencing. A real-time qPCR was then performed using the microfluidic-based Fluidigm 48.48 Dynamic arrays.

Results: We obtained 17 ovaries and 13 testes at various stages of sexual maturation. Of the 37 genes that were tested, 32 (86\%) showed sexually dimorphic expression. These genes included sex-related genes, sox9, wt1, amh, $n r 5 a 2, d m r t 1$ and nrob1, which showed testis-enhanced expression similar to other vertebrate species. Known male- and female-enhanced germ cells markers, which were established from studies in other species, similarly showed testis- and ovary-enhanced expression, respectively, in the Asian seabass. Three pro-Wnt signaling genes were also upregulated in the ovary, consistent with existing studies that suggested the role of Wnt signaling in ovarian differentiation in teleosts and mammals. The expression patterns of genes involved in steroidogenesis, retinoic acid metabolism, apoptosis and NF-KB signaling were also described. We were able to classify gonads according to sex and gonadal maturation stages, based on their small-scale transcriptomic profiles, and to uncover a wide variation in expression profiles among individuals of the same sex.

Conclusions: The analysis of a selected set of genes related to reproduction and in sufficient number of individuals using a qPCR array can elucidate new insights into the molecular mechanisms involved in Asian seabass gonad development. Given the conservation of gene expression patterns found in this study, these insights may also help us draw parallels with other teleosts.

Keywords: $\mathrm{qPCR}$, Barramundi, Maturation, Sex, Gene expression

\footnotetext{
* Correspondence: laszlo@tll.org.sg

'Equal contributors

'Reproductive Genomics Group, Temasek Life Sciences Laboratory, 1

Research Link National University of Singapore, Singapore 117604, Singapore

${ }^{2}$ Department of Biological Sciences, National University of Singapore, 21

Lower Kent Ridge Rd, Singapore 119077, Singapore

Full list of author information is available at the end of the article
} 


\section{Background}

The Asian seabass (Lates calcarifer), which is also commonly known as barramundi, is a protandrous hermaphrodite. Typically, this perciform teleost matures as a functional male at 2-4 years of age and subsequently changes sex to female at subsequent spawning seasons [1-3]. Due to this process, Asian seabass females are generally larger than males.

Primary females that do not appear to go through the male phase, as well as males that do not undergo sex change, may also exist, and these possibilities have been inferred from the presence of young, small-sized females and old males, respectively $[1,3]$. In addition, despite the late sexual maturation age of the species, early testis differentiation can be found in Asian seabass that are grown in intensive freshwater recirculation systems at as early as 9 months old [4].

Although the development of Asian seabass gonads has been well studied morphologically and histologically, the underlying molecular data regarding their development is lacking [1-4]. This lack is despite the identification of several Asian seabass orthologs from the NCBI nucleotide database, including the ovarian aromatase, follistatin and vitellogenin, which are conserved in several teleost species. Next-generation sequencing technologies have only been recently used to provide functional genomics analyses of other aspects of the Asian seabass biology, such as temperature adaptation and the response to stress $[5,6]$.

To date, several important genes have been found to be required for testis or ovary differentiation in a number of model organisms. These sex-related genes include sox9, wt1, amh, nr5a2, dmrt1 and nrOb1, which produce proteins that are required for testis differentiation and have enhanced expression in the testis of several vertebrate species (see Additional file 1: Table S1 for full gene names) [7-13]. For ovarian differentiation, several studies have suggested the role of Wnt signaling in both mammals and teleosts [14-17].

Genes that are involved in steroidogenesis also play a key role in gonad differentiation because these genes lead to the production of sex steroids. Several studies have shown that artificial sex reversal can be easily induced in teleosts through the application of hormones or endocrinedisruptors $[18,19]$. Several steroidogenic genes, such as the ovarian aromatase (cyp19a1) and 11ß-hydroxgenase (cyp11c1), tend to show sexually dimorphic expression in teleosts [9,20-23]. Other than sex steroids, recent studies of the Japanese flounder (Paralichthys olivaceus) and pejerrey (Odontesthes bonariensis) have also shown that cortisol may (a steroid hormone) have a role in testis differentiation [24,25].

Germ cells are key components of the gonads, and their numbers in the early teleost gonads have been suggested to affect sex determination [26]. Several germ cell markers, such as piwill in the zebrafish (Danio rerio) and sycp in the medaka (Oryzias latipes), displayed sexually dimorphic expression in the gonads $[27,28]$. This observation could be due to the structural functions that some germ cell markers have in specific germ cell types, such the role of ODF proteins in forming the cytoskeletal structure of the human sperm tail [29]. Germ cell markers can also play other non-structural roles, such as sperm receptors (zona pellucida genes) or the maintenance of transposon silencing in the germline (piwi-like genes) [30,31]. In addition to key sex-related genes, steroidogenic genes, Wnt signaling genes and germ cell markers, other genes that are involved in retinoic acid signaling, apoptosis and NF- $\mathrm{KB}$ signaling have also been implicated in teleost sex differentiation [32,33].

Based on our current state of knowledge regarding sex differentiation, we shortlisted genes that are involved in the pathways or functions that have been described above for gene expression analysis. In this study, we generated a set of real-time qPCR primers for 37 genes and performed gene expression profiling of Asian seabass testes and ovaries on a mid-throughput microfluidic-based qPCR platform. Our analysis showed that several genes with known reproductive function(s) (e.g., Wnt pathway genes and germ cell markers) exhibited expression patterns in the Asian seabass that were similar to those of other teleosts. We were also able to classify gonads according to sexual maturation status by hierarchical clustering analysis using expression profiles of these genes. In addition, the expression analysis revealed interesting aspects of the Asian seabass gonads and uncovered new insights into their sexual maturation.

\section{Methods}

\section{Fish}

Adult Asian seabass individuals that ranged from $44-65$ $\mathrm{cm}$ standard length were collected from the Marine Aquaculture Centre of the Agri-Food and Veterinary Authority of Singapore. The fish were reared and maintained according to protocols that were approved by the AVA Institutional Animal Care and Use Committee (approval number: AVA-MAC-2011-01). The fish were sacrificed, and gonads were collected for histology and RNA extraction.

\section{RNA isolation and CDNA synthesis}

Total RNA was extracted from the gonads using the RNeasy Mini Kit (Qiagen). For the real-time qPCR experiment, RNA quality and quantity were assessed using agarose gel electrophoresis and a NanoDrop spectrophotometer, respectively. Total RNAs were reverse transcribed using an iScript cDNA Synthesis Kit (Bio-Rad Laboratories) following the manufacturer's instructions. 


\section{Histology and staging of gonads}

Gonads were fixed in 10\% formalin overnight at room temperature. After dehydration by ethanol, samples were embedded in plastic resin (Leica Biosystems), and then serial cross-sections of 5-10 $\mu \mathrm{m}$ were cut using a microtome (Leica Biosystems) and dried on slides at $42^{\circ}$ $\mathrm{C}$ overnight. The sections were stained with hematoxylin and eosin and then mounted in Permount (Thermo Fisher Scientific).

The Asian seabass gonads were classified according to sexual maturation status as described by Guiguen and colleagues [2]. We obtained 17 ovaries, with four at the F1 stage, seven at the F3 stage and six at the F4 stage, as well as 13 testes, with three at the M1 stage, two at the M2 stage, six at the M3 stage and two at the M4 stage (Table 1, see Additional file 2: Figure S1 for the gonad histology).

\section{Isolation, cloning and sequencing of genes with sex-related function}

Partial coding sequence of Asian seabass genes with sexrelated function were obtained using degenerate primerbased amplification and cloning. Several known homologous cDNAs from other teleost species were clustered using ClustalW, and degenerate primers were designed at conserved regions using the software Primer Premier v5.0 (Premier Biosoft) [34]. PCR was then performed on pooled cDNA, which originated from Asian seabass testes and ovaries. The degenerate primer sequences and annealing temperatures that were used for PCR are listed in Additional file 3: Table S2. The PCR products were run on a $2 \%$ agarose gel and stained using GelStar nucleic acid gel stain (Lonza). The band of the expected size was cut from the gel, and then the amplified product was isolated using the QIAquick Gel Extraction Kit (Qiagen) and cloned into the pGEM-T Easy Vector (Promega). The validity of the sequence and the identification of the Asian seabass orthologous gene were completed by running the BLAST algorithm (blastn) against the NCBI RefSeq database (Additional file 1: Table S1).

\section{Transcriptome sequencing}

Total RNAs, which were obtained from Asian seabass testes and ovaries, were depleted of ribosomal RNA using a RiboMinus Eukaryote Kit for RNA-seq (Invitrogen) and verified using an Agilent 2100 Bioanalyzer. The rRNAdepleted total RNA was sent to a service provider for transcriptome sequencing on a SOLiD 3+ platform (Applied Biosystems). Similarly, another rRNA-depleted sample, which was pooled from RNA that was extracted from various Asian seabass organs, was sent to another service provider for transcriptome sequencing on a 454 FLX Titanium platform (Roche). Reads that were obtained from SOLiD 3 + and 454 sequencing were assembled de novo using the programs Velvet, CLC Genomics Workbench (CLC Bio) and Sequencher (Gene Codes) [35]. The reads and assembled transcriptome have been deposited into the NCBI SRA and TSA databases, respectively [SRA accession numbers SRR944005, SRR944006 and SRR949061; TSA accession numbers GAML01000000 and GAMU01000000].

\section{Real-time qPCR}

Real-time qPCR was performed using a BioMark ${ }^{\mathrm{TM}} \mathrm{HD}$ system (Fluidigm Corporation). cDNA from Asian seabass testes and ovaries were used for specific target amplification using the TaqMan PreAmp Master Mix (Life Technologies) and loaded onto Fluidigm's Dynamic Array Integrated Fluidic Circuits (IFC) according to Fluidigm's EvaGreen DNA Binding Dye protocols. The gene symbols, their corresponding accession numbers and gene names are listed in Additional file 1: Table S1, and the primer sequences that were used are listed in Additional file 4: Table S3.

Four 48.48 Dynamic Array IFC plates were used to analyze the expression levels of selected genes in Asian seabass testes and ovaries. Triplicates were analyzed for each biological sample, and four samples were used as inter-plate controls.

Eight genes (18 s, bactin, efla, gapdh, rpl8, tuba, catD and $u b q$ ) were included into the qPCR array to select for endogenous reference genes using the geNorm algorithm

Table 1 Classification of gonadal samples based on histology

\begin{tabular}{llllr}
\hline Sex type & Index & Gonadal maturation stage & Histological criterion & Number of samples \\
\hline Female & F1 & Pre-vitellogenesis & Mostly pre-vitellogenic oocytes & 4 \\
& F3 & Vitellogenesis & Vitellogenic oocytes more than half of section & 7 \\
Male & F4 & Atretic & Presence of atretic oocytes & \\
& M1 & Testis gonia & Mostly gonia & \\
& M2 & Spermatogenesis & Mostly spermatocytes and spermatids & 6 \\
& M3 & Spermiation & Mostly spermatozoa & \\
& M4 & Post-spawning & Absence of spermatozoa in testicular lobules & \\
& Total number of samples & & \\
\end{tabular}


[36]. Of these genes, $r p l 8, e f 1 a$ and $u b q$ had the highest gene expression stability and were used as reference genes (see Additional file 5: Figure S2 for the stability values).

\section{Results and discussion}

The vast majority of genes showed sexually dimorphic gene expression pattern between male and female gonads

Several genes, which have important reproductive functions in mammals and other vertebrates, have been included in this qPCR array. Of the 37 genes chosen in this study (not including the eight reference genes), 86\% $(32 / 37)$ of these genes showed sexually dimorphic expression ( $\mathrm{p}$-value $<0.05$ and fold-change $\geq 1.5$ or $\leq-1.5$ ) when ovaries of F3 and F4 stages were compared against testes of M3 and M4 stages (Table 2). The expression of 25 of these genes was enhanced in testes, with 13 genes showing more than 10-fold upregulation when compared with ovaries. In contrast, seven genes were found to have ovary-enhanced expression, of which $z p 2$ showed the highest upregulation (7.1-fold) in ovaries compared with testes.

\section{Conserved expression patterns of sex-related genes and pathways in the Asian seabass with other teleosts and even mammals \\ Known sex-related genes}

Among the genes in the array, there were genes with "pro-male" functions, such as amh, sox 9 , dmrt1, wt1, $n r 5 a 2$ and $n r 0 b 1$, which displayed conservation of testisenhanced expression in the Asian seabass that was similar to other vertebrates (Table 2). For example, in humans, anti-Müllerian hormone (AMH), which is produced by fetal Sertoli cells, is responsible for the regression of Müllerian ducts during testis differentiation, and its mutation results in the presence of uteri and Fallopian tubes in males [37]. Amh also inhibits follicular growth and aromatase expression in mouse ovaries [38,39]. In teleosts, amh displayed a higher expression in zebrafish testes than in ovaries, and likewise, we detected a strong testis-enhanced expression of amh (19.7-fold upregulation) in the Asian seabass [9,21]. Similarly, sox9, dmrt1, $w t 1, n r 5 a 2$ and $n r O b 1$ were also found to have increased expression in Asian seabass testes compared with ovaries, and earlier, their orthologs were shown to have roles in testis development in other teleosts and even in mammals [7-13].

\section{Germ cell markers}

We have also included several germ cell markers in this array. One of these markers, piwill, has a role in maintaining transposon silencing in the germline genome and has higher expression in testes of adult zebrafish compared with the ovaries $[27,30]$. Similarly, piwil1 was found to have higher expression in Asian seabass testes (Table 2). Another set of germ cell markers, Tudor domain containing proteins or Tdrds, are associated with the nuage or germinal granules of germ cells and have been found to be highly expressed in adult mouse testes [40]. In our array, $t d r d 1$ and $t d r d 7$ also showed testis-enhanced expression in the Asian seabass.

The other four germ cell markers, odf3, sycp3l, sept6 and sept7, are known to be expressed in the spermatids and spermatozoa of other teleost and mammalian species. ODF proteins form the main cytoskeletal structure of the human sperm tail and are found to be essential for male fertility in the zebrafish $[29,41]$. Because of this structural function, odf 3 expression levels had the highest fold difference (2,628-fold) in Asian seabass testes compared with ovaries (Table 2). SCP3 is essential in meiosis progression and in the formation of spermatozoa in mice [42]. In the medaka, sycp has been shown to have higher expression in testes than ovaries, and likewise, we detected a 25.8-fold upregulation in Asian seabass testes (Table 2) [28]. Septins are a family of GTP-binding proteins that forms a component of the cytoskeleton in eukaryotes [43]. These proteins have been shown to be required for sperm development in mouse and have sexually dimorphic expression in zebrafish gonads [22,44]. In Asian seabass, sept6 and sept7 have 7.5-fold and 1.5-fold higher expression in testes compared with ovaries, respectively (Table 2). Additionally, the germ cell markers that we have investigated have displayed conservation in gene expression patterns with other vertebrate species.

\section{Steroidogenesis-related genes}

In teleosts, 17 $\beta$-estradiol (E2) and 11-ketotestosterone $(11-\mathrm{KT})$ are the key female and male hormones, respectively, and sex differentiation can be easily influenced by steroids or endocrine-disruptors, which result in sex reversal $[18,19,45,46]$. Both E2 and 11-KT are synthesized from testosterone, and cyp11c1 converts testosterone to 11-KT [45]. Therefore, cyp11c1 has an important role in testicular development, and this role is reflected in its testis-enhanced expression in teleosts, including the European seabass (Dicentrarchus labrax) and zebrafish $[20,22]$. Accordingly, in the Asian seabass, cyp11c1 has over 300-fold upregulation in the testis (Table 2).

In contrast, cyp19a1a, or ovarian aromatase, is involved in the conversion of testosterone to E2. During zebrafish gonadal transformation from juvenile ovaries to testes, cyp19a1a is downregulated, whereas its expression remains high in adult ovaries $[9,21,23]$. The overexpression of aromatase can also result in ovary development in genetically male chicken embryos [47]. However, contrary to our expectation, an upregulation of gonadal aromatase was found in Asian seabass testes compared 
Table 2 Genes that were analyzed between Asian seabass testes (M3 and M4) and ovaries (F3 and F4) and classified according to functions and pathways

\begin{tabular}{|c|c|c|c|}
\hline Gene symbol & Accession & Fold-change (Ovary vs. Testis) & $\mathrm{p}$-value \\
\hline \multicolumn{4}{|c|}{ Known sex-related } \\
\hline sox9 & KF444460 & -51.8 & 4.95E-04 \\
\hline wt1 & KF444464 & -25.0 & 0.024 \\
\hline$a m h$ & GAMU01071817 & -19.7 & 9.49E-10 \\
\hline$n r 5 a 2$ & KF444453 & -15.2 & 0.007 \\
\hline$d m r t 1$ & KF444450 & -7.8 & 0.000 \\
\hline nrob1 & KF444458 & -7.6 & 0.013 \\
\hline \multicolumn{4}{|c|}{ Germ cell markers } \\
\hline odf3 & GAMU01119126 & -2628.1 & $2.64 \mathrm{E}-13$ \\
\hline syср31 & GAML01036838 & -25.8 & 1.16E-06 \\
\hline tdrd7 & GAML01004579 & -15.4 & 1.37E-08 \\
\hline piwil1 & GAML01007253 & -10.6 & 4.26E-10 \\
\hline sept6 & KF444459 & -7.5 & 2.27E-10 \\
\hline vasa & KF444462 & -3.2 & $1.18 \mathrm{E}-04$ \\
\hline tdrd1 & GAML01005267 & -2.2 & 0.002 \\
\hline sept7 & GAML01001618 & -1.5 & 0.026 \\
\hline$z p 2$ & KF444465 & 7.1 & 0.003 \\
\hline \multicolumn{4}{|c|}{ Steroidogenesis } \\
\hline cyp11c1 & KF4444477 & -377.8 & $1.56 \mathrm{E}-15$ \\
\hline$h s d 3 b$ & KF444455 & -42.1 & 0.038 \\
\hline hsd11b2 & KF444456 & -12.8 & 0.025 \\
\hline cyp19a1 & AY684256.1 & -5.5 & 1.47E-04 \\
\hline cyp17a1 & KF444448 & -5.0 & 0.005 \\
\hline hsd17bl & KF444457 & -4.2 & 0.786 (N.S.) \\
\hline \multicolumn{4}{|l|}{ Wnt signaling } \\
\hline fox 12 & KF444454 & -4.9 & 0.434 (N.S.) \\
\hline $\operatorname{axin} 1$ & KF444443 & -3.1 & 0.242 (N.S.) \\
\hline ctnnbip 1 & KF444446 & 1.5 & 0.072 (N.S.) \\
\hline$c k 2 a$ & KF444444 & 2.0 & 0.042 \\
\hline ctnnbl & KF444445 & 3.5 & 0.001 \\
\hline$d v / 2$ & KF444451 & 4.5 & 0.001 \\
\hline \multicolumn{4}{|c|}{ Retinoic acid signaling } \\
\hline cyp26b1 & KF444449 & -36.3 & 5.61E-06 \\
\hline stra6 & GAML01004693 & 1.3 & 0.079 (N.S.) \\
\hline сур26a1 & GAML01005182 & 1.8 & 0.030 \\
\hline \multicolumn{4}{|l|}{ NF-KB-related } \\
\hline$n f k b 2$ & GAMU01013914 & -3.1 & 2.74E-04 \\
\hline nkap & GAML01003947 & -3.0 & 0.001 \\
\hline \multicolumn{4}{|l|}{ Apoptosis } \\
\hline $\operatorname{tp} 53$ & KF444461 & 2.0 & 0.020 \\
\hline \multicolumn{4}{|l|}{ Others } \\
\hline esrn & KF444452 & -25.1 & $6.68 \mathrm{E}-05$ \\
\hline
\end{tabular}




\begin{tabular}{|c|c|c|c|}
\hline ar & KF444442 & -4.9 & $2.29 \mathrm{E}-06$ \\
\hline psap & GAML01003564 & -2.1 & 0.001 \\
\hline$v \operatorname{tgr}$ & KF444463 & 2.9 & 0.009 \\
\hline
\end{tabular}

N.S. - Not significant (fold-change $<1.5$ or $>-1.5$ or $p$-value $\geq 0.05$ ).

with ovaries (Table 2). One possible explanation is that, although cyp19a1 was overexpressed by 5.5 -fold in the testis, cyp11c1, which is the gene that regulates the 11KT level, was overexpressed by more than 300-fold. Given that both cyp19a1 and cyp11b act on the same precursor, which is testosterone, this result may indicate that estrogen levels may remain low relative to those levels of androgens in males. In addition, Cyp19a1 may also be regulated at the post-transcriptional level in the seabass gonads. It was also recently found that, in some cichlid lineages, both the ovarian and brain aromatases can have testicular function, and as a result, the sex steroid pathway has been suggested to be less conserved among teleosts [48]. Nevertheless, the unexpected testis-enhanced expression of cyp19a1 in the Asian seabass is worth further investigation in the future.

In recent years, cortisol has been shown to be involved in teleost sex differentiation. For example, in the Japanese flounder (Paralichthys olivaceus) and in pejerrey (Odontesthes bonariensis), elevated cortisol levels that are caused by increased temperature can result in female-to-male sex reversal $[24,25]$. Therefore, in this qPCR array, we have also included two genes that are involved in the metabolism of cortisol, which is also an important component of the stress response in fish [49]. Although the interrenal tissues of the head kidney are known to be the major site of cortisol production in teleosts, it is, nevertheless, worthwhile to investigate the gonadal expression of the two genes $h s d 11 b 2$ and $h s d 3 b$ [50].

Hsd11b2 plays a role in the conversion of the physiologically active cortisol to inactive cortisone [51]. It has been suggested that 11ß-hydroxysteroid dehydrogenases protect the teleost gonads from the inhibitory effects of cortisol, such as the inhibition of testicular androgen production [52,53]. In contrast, $h s d 3 b$ is involved in the early steps of the steroidogenic pathway, which results in the production of not only glucocorticoids but also mineralocorticoids and sex steroids [54]. The $h s d 3 b$ gene was also found to be expressed in both the interrenal tissues of the head kidney and in the gonads of zebrafish [54].

In the Asian seabass, both genes were overexpressed in testes compared with ovaries. The gene of the cortisolproducing enzyme $h s d 3 b$ displayed over 40 -fold upregulation, whereas the gene of the cortisol-degrading enzyme, hsd11b2, exhibited 12.8-fold overexpression
(Table 2). Therefore, cortisol might also have reproductionrelated roles in the Asian seabass because of the sexually dimorphic expression of both genes.

\section{Wnt signaling pathway}

Canonical Wnt signaling has been known to be involved in mammalian ovary development, and the overexpression of WNT4 in humans has been associated with XY sex reversal $[15,16,55]$. Similarly, Wnt signaling has already been implicated in a reproductive role in teleosts, including the black porgy (Acanthopagrus schlegeli), rainbow trout (Oncorhynchus mykiss) and zebrafish $[14,17,56,57]$. It has been further shown that Wnt signaling could promote ovarian differentiation through the upregulation of gonadal aromatase $[56,58]$.

Among the sexually dimorphic genes in the Asian seabass, there were three members of the Wnt family: $\beta$-catenin 1 (ctnnb1), $d v l 2$ and $c k 2 a$. $\beta$-catenin is the central molecule in the canonical Wnt signaling pathway. In the absence of Wnt ligand binding, cytoplasmic $\beta$-catenin is degraded by the ubiquitin-proteosome pathway and prevented from entering the nucleus and associating with Lef1 to activate Wnt target genes $[59,60]$. Dishevelled is a positive transducer of Wnt signals and functions to activate both canonical and non-canonical Wnt signaling pathways [61]. In mouse, $C k 2$ phosphorylates $\beta$-catenin and, therefore, protects $\beta$-catenin from being degraded by the ubiquitinproteosome pathway [62]. Ck2 has also been found to be activated by Wnt3a [63]. All three "pro-Wnt signaling" genes were upregulated in Asian seabass ovaries compared with testes, and this result suggested that, similar to mammals and other teleost species, the Wnt signaling pathway has a pro-female function in this species as well (Table 2).

\section{Retinoic acid (RA) signaling pathway}

RA is catabolized by both Cyp26a1 and Cyp26b1 [64]. In mice, Cyp26b1 is required to retard or block germ cells from entry into meiosis in the testes and to prevent the apoptosis or conversion of the male germ cell fate $[65,66]$. Cyp26b1 expression in mice is also thought to be activated by Sox 9 and Sf1 in the testes and inhibited by Foxl2 in the ovaries [67]. Although little is known regarding the role of Cyp26a1 in reproduction, the exposure of 
mice to RA resulted in the apoptosis of spermatogonia and in the increased expression of this gene [68].

In the Asian seabass, cyp26b1 was strongly upregulated in testes compared with ovaries, whereas there was only a slight downregulation of cyp26a1 (Table 2). Thus, the sexually dimorphic expression of cyp26b1 and cyp26a1 suggest the possibility of retinoic acid signaling involvement in testes development in the Asian seabass.

\section{Genes with apoptosis-related function}

During gonadal transformation in zebrafish, apoptosis is required to remove the unwanted female cells to clear up space' for the developing testicular cells [69,70]. In zebrafish, the main function of fancl is to ensure the survival of female germ cells. As a result, its mutation causes zebrafish to develop as males, and its effects can be rescued through the mutation of the tp53 tumor suppressor gene, which is a well-known pro-apoptotic gene [71,72]. Our results demonstrated that tp53 was upregulated by two-fold in Asian seabass ovaries compared with testes. It is possible that this result is due to the atresia that occurs in the F4 ovaries and that the testes that are not undergoing transformation to ovaries are unlikely to have higher levels of apoptosis.

\section{NF-KB pathway}

NF- $k B$ is a ubiquitous transcription factor that has been known to be involved in several biological processes, which include the immune response [73-75]. In mammals, NF- $\mathrm{kB}$ was shown to interact with SF-1 and to prevent SF-1 from activating Amh, thus inhibiting Amh expression [76]. In the zebrafish, NF-kB has been shown to inhibit apoptosis during gonad transformation, and thereby, to promote female bias [77]. In contrast, NKAP is an activator of NF- $\mathrm{kB}$, is also shown to repress Notch signaling and is necessary for T-cell development $[78,79]$. Although the NF-kB pathway seems to be a "pro-female" pathway, both genes (i.e., $n f k b 2$ and $n k a p$ ) were downregulated in Asian seabass ovaries compared with testes. In the adult gonads of Asian seabass, $N F-\kappa B$ may have additional reproduction-related roles as suggested by the sexually dimorphic expressions of $n f k b 2$ and $n k a p$.

\section{The expression profiles of $\mathbf{3 6}$ genes were sufficient to distinguish between male and female gonad types in the Asian seabass}

A one-way ANOVA was performed, and all the genes that were tested, except foxl2 and the reference genes, showed significant differences in their expression levels across the various gonad types ( $p$-value $<0.05$ ). The expression profiles of these 36 genes were used to generate a hierarchical clustering map using the Partek Genomics v6 Suite (Partek Inc.) software (Figure 1).

From the hierarchical clustering map, we can observe that the ovaries and testes samples were clustered into two separate primary clades, and within the ovary clade, the F3 ovaries were clustered into their own sub-clade (Figure 1). This result indicated that the F3 ovaries had a distinct gene expression profile from that of ovaries from other stages. In particular, although F3 and F4 ovaries looked similar under histology, $70 \%$ of the tested genes (26/37) were differentially expressed between these two stages.

The genes that were upregulated in F4 ovaries compared with F3 ovaries included germ cell markers, such as vasa, piwill, tdrd 1 and sycp3l; testis-enhanced genes, such as dmrt1, amh, nrOb1; Wnt signaling member genes, such as axin1, dvl2, ctnnb1, ck2a and ctnnbip1; apoptosisrelated genes, such as tp53 and genes that are involved in the retinoic acid pathway (cyp26a1 and stra6) (Additional file 6: Table S4). The key histological feature that separates F3 and F4 ovaries were the presence of atretic oocytes (Additional file 2: Figure S1). The pro-apoptotic gene tp53 was upregulated by 2.6-fold in the F4 ovary compared with the F3 ovary, and this upregulation may be a consequence of atresia in the F4 ovary. In addition, the upregulation of 24 genes and the downregulation of only three genes in F4 ovaries compared with F3 ovaries suggest that the former may be more transcriptomically active than the latter.

We can also observe from the hierarchical clustering map that individual differences in gene expression exist within each group, which shows the complexity and variability in the process of gonad development. Studies regarding gonad differentiation in the zebrafish have also reported such a trend $[80,81]$. The individual variability of gonad development hence dictates that more biological samples would be required to obtain a more representative result for any sex-related experiment. In this sense, the use of a 48.48-type GPCR array would prove useful because this method allows the analysis of only the important genes in several individuals (up to 13) in parallel. This result also demonstrated that the analysis of a moderate number of well-selected genes that are relevant to the area of interest (reproduction in our case) and in a sufficient number of individuals could be a powerful tool for improving our understanding of the molecular regulation of these complex processes.

\section{Female-like expression levels of $a m h$ and germ cell markers in $\mathrm{M} 1$ testes}

Similarly, from the hierarchical clustering map, we can see that, within the testes clade, the inactive M1 testes were clustered in their own sub-clade. M1 testes contain predominately gonia, and these cells are inactive and incapable of spawning. Gene expression analysis showed that these M1 testes have female-like expression levels of the testis-enhanced germ cell markers, odf3, sycp3l, septin6 and $t d r d 7$, as well as amh (Figure 2). This result 


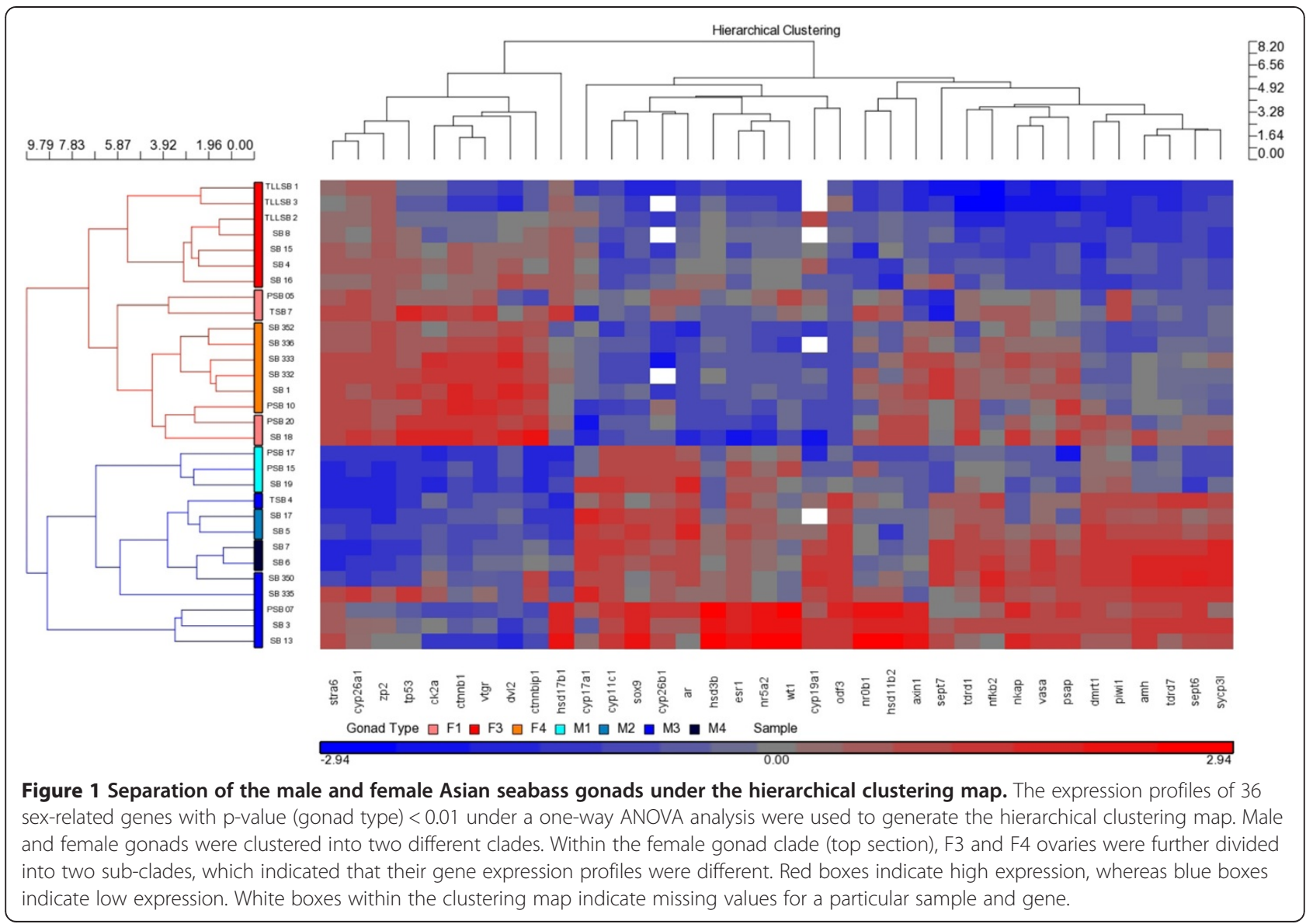

is likely to be a direct consequence of the lack of spermatids and spermatozoa in the M1 testes because these germ cell markers, odf3, sycp3l and septin6, are involved in the formation of the spermatozoa, as shown in other species, whereas $t d r d 7$ is associated with male germ cells $[29,40,42,44]$. In the case of $a m h$, it has been demonstrated in the black porgy (Acanthopagrus schlegeli) that active testes have higher expression levels than inactive ones, which potentially indicates that a higher level of amh might be required to initiate spermatogenesis [82].

\section{Increased variation of testicular $z p 2$ expression because} of the presence of pre-vitellogenic oocytes in some of the Asian seabass testes

Zona pellucida glycoproteins, which are composed of $z p 1, z p 2$ and $z p 3$ in mammals, are found on the extracellular matrix of oocytes and serve as receptors for the binding of sperm; their orthologs were found in fish oocytes $[31,83,84]$. As expected, in the Asian seabass, $z p 2$, which is an oocyte marker, had a 7.1-fold upregulation in the ovaries compared with testes. However, although $z p 2$ expression showed consistently high expression values across all the female gonads, there was a high variation among the male gonads, with some M3-testes showing increased $z p 2$ transcript levels compared with the other male gonads (Figure 3 ). The high $z p 2$ expression variation may be related to the presence of pre-vitellogenic oocytes that can be found in the histological sections of some M3 testes (Figure 4), which confirms previous descriptions by others [2].

The sexually dimorphic expression of cyp $11 c 1$ and esr 1 is independent of the gonadal maturation status of gonads Two of the studied genes, cyp11c1 and esr1, were found to be expressed in a distinctly sexually dimorphic manner between the gonads of the male and female sex, regardless of their sexual maturation stage (Figure 5). Both genes showed testis-enhanced expression, even at the inactive M1 and M2 stages, whereas their expression levels were uniformly low across the F1, F3 and F4 ovaries.

This result indicated that the consistently testisenhanced expression of cyp11c1 and esr 1 could be good expression markers for testes and suggested the importance of the two genes in maintaining the male sex of the gonad. Although the upregulation of esr 1 in testes may seem counter-intuitive, given the importance of estrogens in ovary development, estrogen receptors (ER) have been suggested to have a role in the control of testicular 

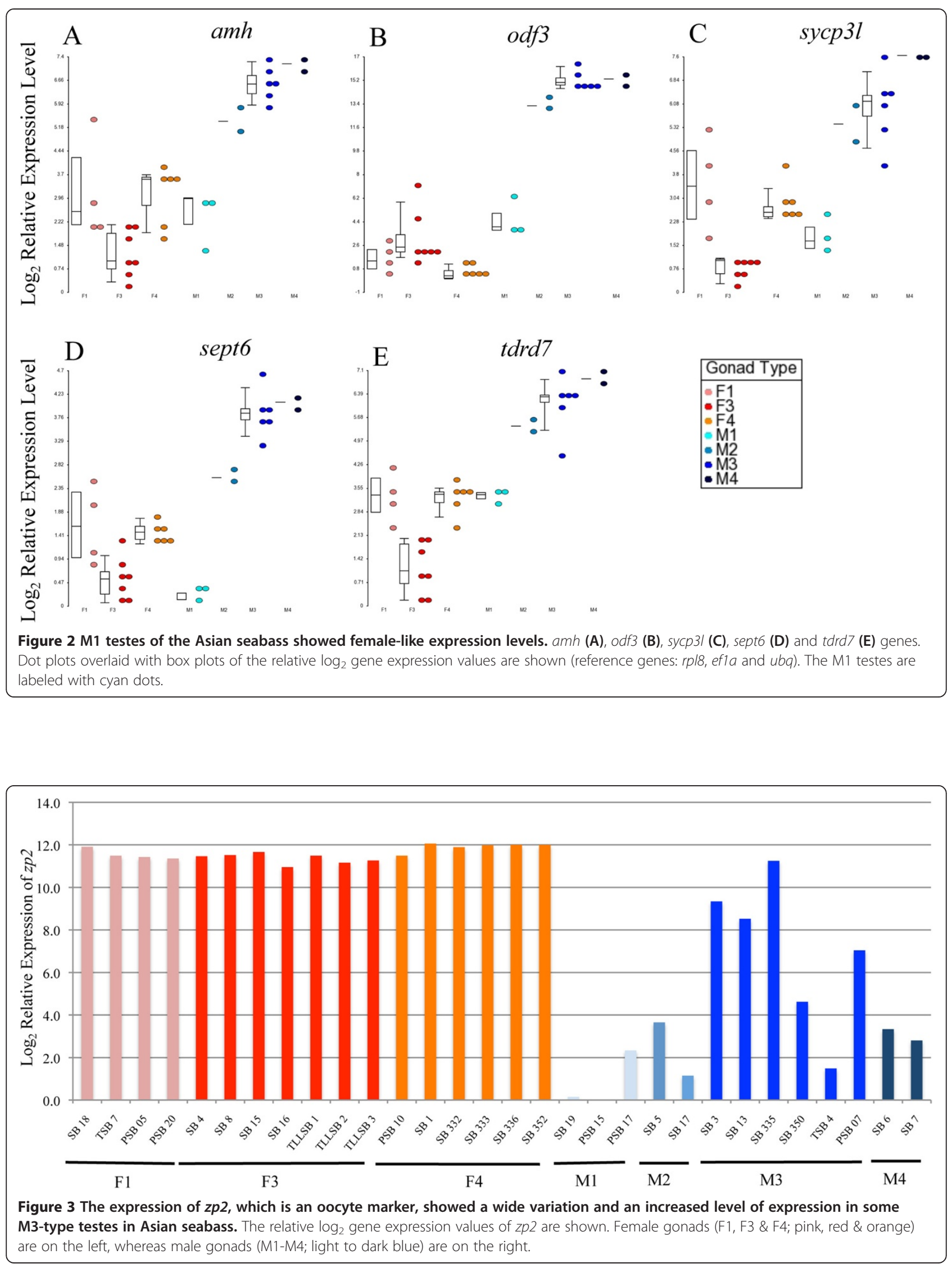


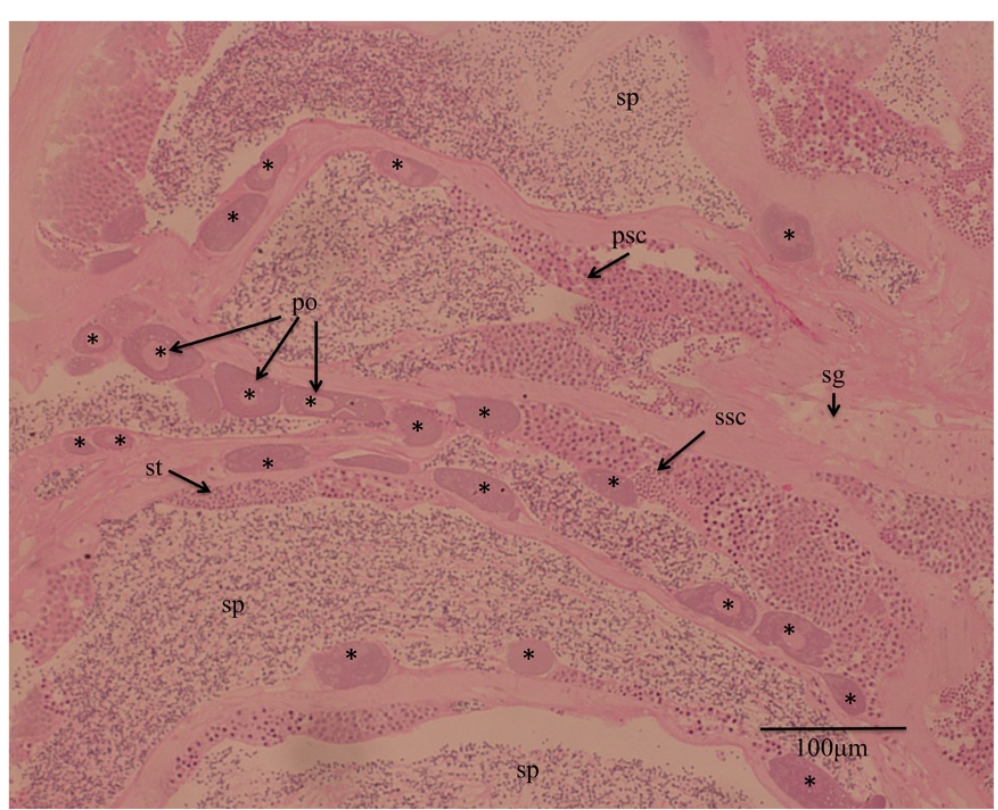

Figure 4 Pre-vitellogenic oocytes can be found lining the tubular walls in the M3 testis of an Asian seabass individual (SB335).

Abbreviations: po - previtellogenic oocytes; sp - spermatozoa; st - spermatids; ssc - secondary spermatogonia; psc - primary spermatogonia. Pre-vitellogenic oocytes are indicated with asterisks.

function because both $E R a$ and $E R b$ are expressed in the human testis [85]. Similarly, we do find two paralogs of estrogen receptors in the Asian seabass, but have only analyzed the expression pattern of esrl in this study. A higher testicular expression level of esr 1 compared with that of the ovary was also observed in both the medaka and sea bream (Sparus aurata) [86-88].

\section{Zebrafish as a model for reproductive studies in Asian} seabass

We propose that the zebrafish can be a model for Asian seabass reproductive studies and possibly even for other sequential hermaphrodites because of the conserved role of several sex-related genes that were described in this study.

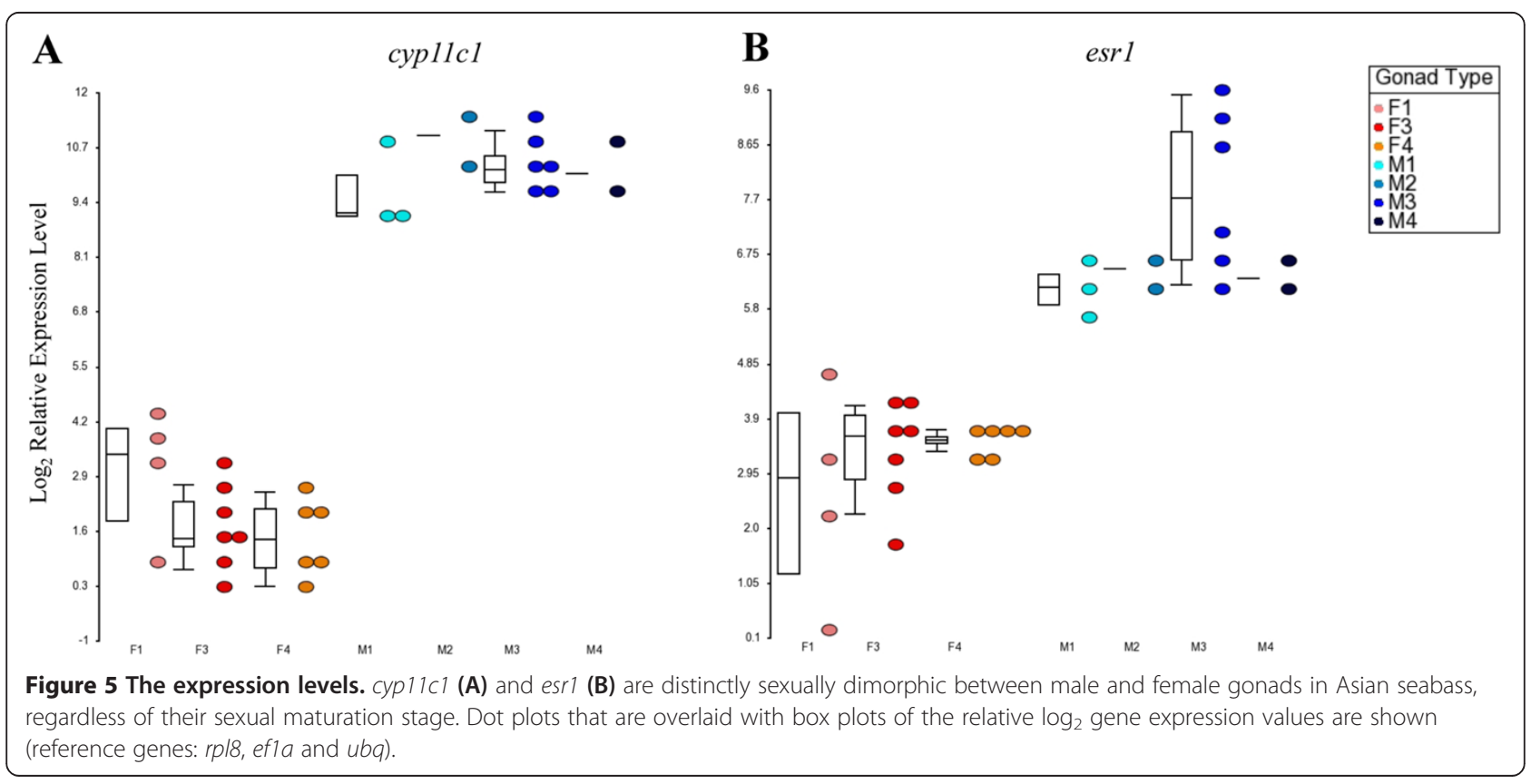


All zebrafish develop a juvenile ovary before future males undergo gonadal transformation to form the testis $[69,70,89]$. Therefore, unlike the Asian seabass, the ovary undergoes gonadal transformation to become the testis, and the process occurs during early differentiation. However, although the zebrafish and Asian seabass systems are developing in the opposite directions (ovary to testis vs. testis to ovary, respectively), we found a largely overlapping set of genes that showed differential expression in the zebrafish and Asian seabass. As such, it seems that the underlying molecular mechanisms that are involved in gonad transformation are conserved in both systems. Therefore, we have developed a working hypothesis for future studies of gonad transformation in the Asian seabass, which are based on the zebrafish model (Figure 6).

In this working model, genes or pathways that are involved in the zebrafish ovary-to-testis transformation are simply "reflected" during Asian seabass testis-to-ovary transformation, in the sense that the same genes and pathways are also involved, except their expression changes in the opposite direction. For example, if a gene or pathway is upregulated during zebrafish ovary-to-testis transformation, then the corresponding ortholog should be downregulated during seabass testis-to-ovary transformation and vice-versa. (Note here that apoptotic genes might be an exception because apoptosis is expected to be higher in the gonad type that will undergo the transformation compared with the other type that will be produced because of the transformation.) Hence, future studies of gonad transformation in Asian seabass can take reference from the well-studied zebrafish model because of the conservation of expression patterns of several genes between the two systems.

\section{Conclusions}

We have generated a platform that allowed us to look at a moderate set of sex-related genes, which were carefully chosen based on earlier data from other species. Our study is the first to report such mid-throughput molecular data regarding gonad maturation in the Asian seabass. The results in our study show that several genes that are known to be involved in reproductive functions are also conserved in their gene expression pattern in the Asian seabass. Our data also indicate that the process of gonad maturation has high individual variability and complexity. The expression patterns of our custom gene set also reflected interesting aspects of the Asian seabass gonads and provided new insights into their sexual maturation and development. In the future, sequencing the complete transcriptome of the species and the use of a whole-transcriptome expression microarray (or RNAseq), together with the working hypothesis that was developed using the zebrafish model, will help
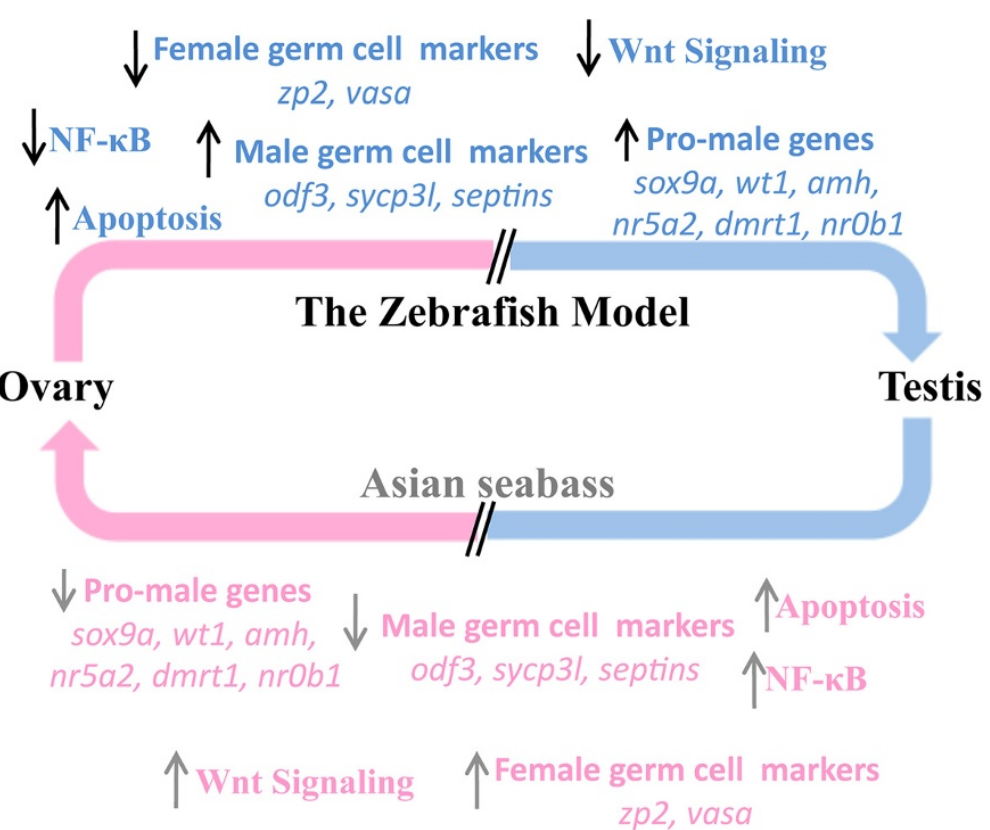

Figure 6 Working hypothesis for the gonad transformation process. All zebrafish develop a juvenile ovary before the future males undergo gonadal transformation to form the testis. Several pathways and genes have been known to be involved in this process. Based on our results, we proposed that the same pathways and genes are also involved in the testis-to-ovary transformation process in the Asian seabass, despite the reversal of direction. The Asian seabass system is, hence, a mirror image of the zebrafish model. The arrows show the observed (zebrafish; top - blue) and/or expected (Asian seabass; bottom - pink) up- or downregulation of the genes or pathways during the gonadal transformation process. 
us further understand the molecular mechanisms that are involved in gonad transformation and also help us draw parallels with other systems. The use of early undifferentiated and transforming gonads in future studies would also be desired to more accurately identify the genes or pathways that are involved in testis and ovary differentiation, respectively.

\section{Additional files}

Additional file 1: Table S1. Gene symbols, with corresponding NCBI accession numbers, and full names of the genes that were analyzed. The identification of the genes that were obtained through this study was performed by running the BLAST algorithm (blastn) in NCBI against the NCBI RefSeq database.

Additional file 2: Figure S1. Ovarian and testicular maturation stages in the Asian seabass that were obtained for this study. The classification of the sexual maturation stages was based on Guiquen et al., Environ Biol Fishes 1994, 39(3):231-247. Abbreviations: po - pre-vitellogenic oocytes; vi - vitellogenic oocytes; ca - cortical alveolus oocytes; ao - atretic oocytes; g - gonia; st - spermatids; sc - spermatogonia; sp - spermatozoa; r.sp residual spermatozoa.

Additional file 3: Table S2. List of degenerate primer sequences and annealing temperatures that were used in cloning of Asian seabass cDNAs.

Additional file 4: Table S3. The list of primer sequences that were used. Additional file 5: Figure S2. Stability values of the candidate reference genes that were obtained using the algorithm GeNorm. The use of three reference genes was optimal for normalization (A), and rp/8, efia and $u b q$ had the highest gene expression stability (B).

Additional file 6: Table S4. The list of differentially expressed genes between Asian seabass F3 and F4 ovaries was optimal for normalization (A), and rpl8, efla and $u b q$ had the highest gene expression stability (B).

\section{Competing interests}

The authors declare that they have no competing interests.

\section{Authors' contributions}

PR cloned the sex-related sequences, designed the GPCR primers, extracted RNA and performed the GPCR array experiment. JHJ collected and classified the gonad samples, generated RNA samples for next-generation sequencing, assembled the transcriptome, performed the data analysis and drafted the manuscript; WCL participated in the sample collection and performed the qPCR; LO conceived and supervised the work and helped to draft the manuscript. All authors read, corrected and approved the final manuscript.

\section{Acknowledgments}

We would like to thank attachment students Husna Fatinah Hussein, Sabeen Khalid and Mark Samson for their assistance in the cloning and histology work. We would also like to thank the staff from the Marine Aquaculture Centre, Agri-Food and Veterinary Authority of Singapore for their assistance in the collection of gonad samples. This study was funded by the Agri-Food and Veterinary Authority of Singapore and by internal grants from the Strategic Research Program of Temasek Life Sciences Laboratory.

\section{Author details}

${ }^{1}$ Reproductive Genomics Group, Temasek Life Sciences Laboratory, 1 Research Link National University of Singapore, Singapore 117604, Singapore. ${ }^{2}$ Department of Biological Sciences, National University of Singapore, 21 Lower Kent Ridge Rd, Singapore 119077, Singapore. ${ }^{3}$ Agri-Food and Veterinary Authority of Singapore, 5 Maxwell Rd, Singapore 069110, Singapore. ${ }^{4}$ School of Biological Sciences, Nanyang Technological University, 50 Nanyang Ave, Singapore 639798, Singapore. ${ }^{5}$ Department of Animal Sciences and Animal Husbandry, Georgikon Faculty, University of Pannonia, H-8360, Keszthely, Hungary. ${ }^{6}$ Present address: National Centre for Biological
Sciences, Tata Institute of Fundamental Research, GKVK, Bellary Road, Bangalore 560065, India.

Received: 29 August 2013 Accepted: 25 December 2013

Published: 9 January 2014

\section{References}

1. Davis T: Maturity and sexuality in barramundi, Lates calcarifer (Bloch), in the Northern Territory and south-eastern Gulf of Carpentaria. Mar Freshw Res 1982, 33(3):529-545.

2. Guiguen Y, Cauty C, Fostier A, Fuchs J, Jalabert B: Reproductive cycle and sex inversion of the seabass, Lates calcarifer, reared in sea cages in French Polynesia: histological and morphometric description. Environ Biol Fishes 1994, 39(3):231-247.

3. Moore R: Natural sex inversion in the giant perch (Lates calcarifer). Mar Freshw Res 1979, 30(6):803-813.

4. Szentes K, Mészáros E, Szabó T, Csorbai B, Borbély G, Bernáth G, Urbányi B, Horváth Á: Gonad development and gametogenesis in the Asian sea bass (Lates calcarifer) grown in an intensive aquaculture system. J Appl Ichthyol 2012, 28(6):883-885.

5. Newton JR, Zenger KR, Jerry DR: Next-generation transcriptome profiling reveals insights into genetic factors contributing to growth differences and temperature adaptation in Australian populations of barramundi (Lates calcarifer). Mar Genomics 2013, 11:45-52.

6. Xia JH, Liu P, Liu F, Lin G, Sun F, Tu R, Yue GH: Analysis of stressresponsive transcriptome in the intestine of Asian seabass (Lates calcarifer) using RNA-Seq. DNA Res 2013, 20:449-460.

7. Kent J, Wheatley SC, Andrews JE, Sinclair AH, Koopman P: A male-specific role for SOX9 in vertebrate sex determination. Development 1996, 122(9):2813-2822

8. Nakamoto M, Suzuki A, Matsuda M, Nagahama Y, Shibata N: Testicular type Sox9 is not involved in sex determination but might be in the development of testicular structures in the medaka, Oryzias latipes. Biochem Biophys Res Commun 2005, 333(3):729-736.

9. Rodríguez-Marí A, Yan Y-L, BreMiller RA, Wilson C, Cañestro C, Postlethwait JH: Characterization and expression pattern of zebrafish anti-Müllerian hormone (amh) relative to sox9a, sox9b, and cyp19a1a, during gonad development. Gene Expr Patterns 2005, 5(5):655-667.

10. Raymond CS, Kettlewell JR, Hirsch B, Bardwell VJ, Zarkower D: Expression of Dmrt1 in the genital ridge of mouse and chicken embryos suggests a role in vertebrate sexual development. Dev Biol 1999, 215(2):208-220.

11. Matsuda M, Nagahama Y, Shinomiya A, Sato T, Matsuda C, Kobayashi T, Morrey CE, Shibata N, Asakawa S, Shimizu N, et al: DMY is a Y-specific DM-domain gene required for male development in the medaka fish. Nature 2002, 417(6888):559-563.

12. Nachtigal MW, Hirokawa Y, Enyeart-VanHouten DL, Flanagan JN, Hammer GD, Ingraham HA: Wilms' Tumor 1 and Dax-1 modulate the orphan nuclear receptor SF-1 in sex-specific gene expression. Cell 1998, 93(3):445-454.

13. Von Hofsten J, Olsson PE: Zebrafish sex determination and differentiation: involvement of FTZ-F1 genes. Reprod Biol Endocrinol 2005, 3:63.

14. Wu GC, Chang CF: wnt4 Is associated with the development of ovarian tissue in the protandrous black porgy, Acanthopagrus schlegeli. Biol Reprod 2009, 81(6):1073-1082.

15. Bernard P, Ryan J, Sim H, Czech DP, Sinclair AH, Koopman P, Harley VR: Wnt signaling in ovarian development inhibits Sf1 activation of Sox9 via the Tesco enhancer. Endocrinology 2012, 153(2):901-912.

16. Jordan BK, Mohammed M, Ching ST, Delot E, Chen XN, Dewing P, Swain A, Rao PN, Elejalde BR, Vilain E: Up-regulation of WNT-4 signaling and dosage-sensitive sex reversal in humans. Am J Hum Genet 2001, 68 (5):1102-1109.

17. Sreenivasan R, Jiang J, Wang X, Bartfai R, Kwan HY, Christoffels A, Orban L: Gonad differentiation in zebrafish is regulated by the canonical Wnt signaling pathway. Biol Reprod 2013. doi:10.1095/ biolreprod.113.110874.

18. Orban L, Sreenivasan R, Olsson P-E: Long and winding roads: Testis differentiation in zebrafish. Mol Cell Endocrinol 2009, 312(1-2):35-41.

19. Devlin $\mathrm{RH}$, Nagahama Y: Sex determination and sex differentiation in fish: an overview of genetic, physiological, and environmental influences. Aquaculture 2002, 208(3-4):191-364

20. Socorro S, Martins RS, Deloffre L, Mylonas CC, Canario AVM: A CDNA for European sea bass (Dicentrachus labrax) 11ß-hydroxylase: Gene 
expression during the thermosensitive period and gonadogenesis. Gen Comp Endocrinol 2007, 150(1):164-173.

21. Wang XG, Orban L: Anti-Mullerian hormone and 11 beta-hydroxylase show reciprocal expression to that of aromatase in the transforming gonad of zebrafish males. Dev Dyn 2007, 236(5):1329-1338.

22. Sreenivasan $R$, Cai M, Bartfai $R$, Wang $X$, Christoffels A, Orban L: Transcriptomic analyses reveal novel genes with sexually dimorphic expression in the zebrafish gonad and brain. PLOS ONE 2008, 3(3):e1791.

23. Jorgensen A, Morthorst JE, Andersen O, Rasmussen LJ, Bjerregaard P: Expression profiles for six zebrafish genes during gonadal sex differentiation. Reprod Biol Endocrinol 2008, 6:25

24. Yamaguchi T, Yoshinaga N, Yazawa T, Gen K, Kitano T: Cortisol is involved in temperature-dependent sex determination in the Japanese flounder. Endocrinology 2010, 151(8):3900-3908.

25. Hattori RS, Fernandino Jl, Kishii A, Kimura H, Kinno T, Oura M, Somoza GM, Yokota M, Strüssmann CA, Watanabe S: Cortisol-induced masculinization: does thermal stress affect gonadal fate in pejerrey, a teleost fish with temperature-dependent sex determination? PloS ONE 2009, 4(8):e6548.

26. Siegfried KR, Nüsslein-Volhard C: Germ line control of female sex determination in zebrafish. Dev Biol 2008, 324(2):277-287.

27. Tan C-H, Lee T-C, Weeraratne SD, Korzh V, Lim T-M, Gong Z: Ziwi, the zebrafish homologue of the Drosophila piwi: co-localization with vasa at the embryonic genital ridge and gonad-specific expression in the adults. Mech Dev 2002, 119(Supplement (0)):S221-S224.

28. Iwai T, Yoshii A, Yokota T, Sakai C, Hori H, Kanamori A, Yamashita M: Structural components of the synaptonemal complex, SYCP1 and SYCP3, in the medaka fish Oryzias latipes. Exp Cell Res 2006, 312(13):2528-2537.

29. Petersen $C$, Füzesi $L$, Hoyer-Fender $S$ : Outer dense fibre proteins from human sperm tail: molecular cloning and expression analyses of two cDNA transcripts encoding proteins of 70 kDa. Mol Hum Reprod 1999, 5(7):627-635.

30. O'Donnell KA, Boeke JD: Mighty piwis defend the germline against genome intruders. Cell 2007, 129(1):37-44.

31. Howes E, Pascall JC, Engel W, Jones R: Interactions between mouse ZP2 glycoprotein and proacrosin; a mechanism for secondary binding of sperm to the zona pellucida during fertilization. J Cell Sci 2001, 114(22):4127-4136.

32. Liew WC, Orbán L: Zebrafish sex: a complicated affair. Brief Funct Genomics 2013. doi: 10.1093/bfgp/elt041.

33. Rodríguez-Marí A, Cañestro C, BreMiller RA, Catchen JM, Yan Y-L, Postlethwait JH: Retinoic acid metabolic genes, meiosis, and gonadal sex differentiation in zebrafish. PLOS ONE 2013, 8(9):e73951.

34. Larkin MA, Blackshields G, Brown NP, Chenna R, McGettigan PA, McWilliam H, Valentin F, Wallace IM, Wilm A, Lopez R, et al: Clustal W and Clustal X version 2.0. Bioinformatics 2007, 23(21):2947-2948.

35. Zerbino DR, Birney E: Velvet: Algorithms for de novo short read assembly using de Bruijn graphs. Genome Res 2008, 18(5):821-829.

36. Vandesompele J, De Preter K, Pattyn F, Poppe B, Van Roy N, De Paepe A, Speleman F: Accurate normalization of real-time quantitative RT-PCR data by geometric averaging of multiple internal control genes. Genome Biol 2002, 3(7):research0034.0031-research0034.0011.

37. Josso N, Belville C, Di Clemente N, Picard J-Y: AMH and AMH receptor defects in persistent Müllerian duct syndrome. Hum Reprod Update 2005 11(4):351-356

38. Durlinger AL, Gruijters MJ, Kramer P, Karels B, Ingraham HA, Nachtigal MW Uilenbroek JT, Grootegoed JA, Themmen AP: Anti-Mullerian hormone inhibits initiation of primordial follicle growth in the mouse ovary. Endocrinology 2002, 143(3):1076-1084.

39. Josso N, Racine C, Di Clemente N, Rodolfo R, Xavier F: The role of anti-Müllerian hormone in gonadal development. Mol Cell Endocrinol 1998, 145(1-2):3-7.

40. Hosokawa M, Shoji M, Kitamura K, Tanaka T, Noce T, Chuma S, Nakatsuji N: Tudor-related proteins TDRD1/MTR-1, TDRD6 and TDRD7/TRAP: Domain composition, intracellular localization, and function in male germ cells in mice. Dev Biol 2007, 301(1):38-52.

41. Hsu C-C, Hou M-F, Hong J-R, Wu J-L, Her G: Inducible male infertility by targeted cell ablation in zebrafish testis. Mar Biotechnol 2010, 12(4):466-478.

42. Yuan L, Liu J-G, Zhao J, Brundell E, Daneholt B, Höög C: The murine SCP3 gene is required for synaptonemal complex assembly, chromosome synapsis, and male fertility. Mol Cell 2000, 5(1):73-83.
43. Estey MP, Kim MS, Trimble WS: Septins. Curr Biol 2011, 21(10):R384-R387.

44. Kissel H, Georgescu M-M, Larisch S, Manova K, Hunnicutt GR, Steller H: The Sept4 septin locus is required for sperm terminal differentiation in mice. Dev Cell 2005, 8(3):353-364.

45. J-F Baroiller YG, Fostier A: Endocrine and environmental aspects of sex differentiation in fish. Cell Mol Life Sci 1999, 55:910-931.

46. Hishida T-O, Kawamoto N: Androgenic and male-inducing effects of 11-ketotestosterone on a teleost, the medaka (Oryzias latipes). J Exp Zool 1970, 173(3):279-283.

47. Lambeth LS, Cummins DM, Doran TJ, Sinclair AH, Smith CA: Overexpression of aromatase alone is sufficient for ovarian development in genetically male chicken embryos. PLOS ONE 2013, 8(6):e68362.

48. Böhne A, Heule C, Boileau N, Salzburger W: Expression and sequence evolution of aromatase cyp19a1 and other sexual development genes in east African cichlid fishes. Mol Biol Evol 2013, 30(10):2268-2285.

49. Mommsen T, Vijayan M, Moon T: Cortisol in teleosts: dynamics, mechanisms of action, and metabolic regulation. Rev Fish Biol Fish 1999, 9(3):211-268.

50. Patrizia Gallo V, Civinini A: Survey of the adrenal homolog in teleosts. Int Rev Cytol 2003, 230:89-187.

51. Nematollahi MA, Van Pelt-Heerschap H, Atsma W, Komen J: High levels of corticosterone, and gene expression of star, cyp17a2, hsd3b, cyp21, hsd 1162 during acute stress in common carp with interrenal hyperplasia. Gen Comp Endocrinol 2012, 176(2):252-258.

52. Consten D, Lambert JGD, Komen H, Goos HJT: Corticosteroids affect the testicular androgen production in male common carp (Cyprinus carpio L.). Biol Reprod 2002, 66(1):106-111.

53. Kusakabe M, Nakamura I, Young G: $11 \beta$-Hydroxysteroid dehydrogenase complementary deoxyribonucleic acid in rainbow trout: cloning, sites of expression, and seasonal changes in gonads. Endocrinology 2003, 144(6):2534-2545.

54. Hsu H-J, Lin J-C, Chung B-C: Zebrafish cyp11a1 and hsd3b genes: Structure, expression and steroidogenic development during embryogenesis. Mol Cell Endocrinol 2009, 312(1-2):31-34

55. Vainio S, Heikkila M, Kispert A, Chin N, McMahon AP: Female development in mammals is regulated by Wnt-4 signalling. Nature 1999, 397(6718):405-409.

56. Nicol B, Yano A, Jouanno E, Guerin A, Fostier A, Guiguen Y: Follistatin is an early player in rainbow trout ovarian differentiation and is both colocalized with aromatase and regulated by the Wnt pathway. Sex Dev 2013, 7:267-276

57. Zhang Y, Li F, Sun D, Liu J, Liu N, Yu Q: Molecular analysis shows differential expression of R-spondin1 in zebrafish (Danio rerio) gonads. Mol Biol Rep 2011, 38(1):275-282.

58. Parakh TN, Hernandez JA, Grammer JC, Weck J, Hunzicker-Dunn M, Zeleznik AJ, Nilson JH: Follicle-stimulating hormone/cAMP regulation of aromatase gene expression requires $\beta$-catenin. Proc Natl Acad Sci USA 2006, 103(33):12435-12440.

59. Aberle H, Bauer A, Stappert J, Kispert A, Kemler R: $\beta$-catenin is a target for the ubiquitin-proteasome pathway. EMBO J 1997, 16(13):3797-3804.

60. Behrens J, Von Kries JP, Kuhl M, Bruhn L, Wedlich D, Grosschedl R, Birchmeier $W$ : Functional interaction of $\beta$-catenin with the transcription factor LEF-1. Nature 1996, 382(6592):638-642.

61. Gao C, Chen Y-G: Dishevelled: The hub of Wnt signaling. Cell Signal 2010, 22(5):717-727

62. Song DH, Dominguez I, Mizuno J, Kaut M, Mohr SC, Seldin DC: CK2 Phosphorylation of the armadillo repeat region of $\beta$-catenin potentiates Wnt signaling. J Mol Biochem 2003, 278(26):24018-24025.

63. Gao Y, Wang H-y: Casein kinase 2 is activated and essential for Wnt/ß-catenin signaling. J Mol Biochem 2006, 281(27):18394-18400.

64. Thatcher JE, Isoherranen N: The role of CYP26 enzymes in retinoic acid clearance. Expert Opin Drug Metab Toxicol 2009, 5(8):875-886.

65. MacLean G, Li H, Metzger D, Chambon P, Petkovich M: Apoptotic extinction of germ cells in testes of Cyp26b1 knockout mice. Endocrinology 2007, 148(10):4560-4567.

66. Bowles J, Knight D, Smith C, Wilhelm D, Richman J, Mamiya S, Yashiro K, Chawengsaksophak K, Wilson MJ, Rossant J, et al: Retinoid signaling determines germ cell fate in mice. Science 2006 312(5773):596-600.

67. Kashimada K, Svingen T, Feng C-W, Pelosi E, Bagheri-Fam S, Harley VR, Schlessinger D, Bowles J, Koopman P: Antagonistic regulation of Cyp26b1 
by transcription factors SOX9/SF1 and FOXL2 during gonadal development in mice. FASEB J 2011, 25(10):3561-3569.

68. Snyder EM, Davis JC, Zhou Q, Evanoff R, Griswold MD: Exposure to retinoic acid in the neonatal but not adult mouse results in synchronous spermatogenesis. Biol Reprod 2011, 84(5):886-893.

69. Maack G, Segner $\mathrm{H}$ : Morphological development of the gonads in zebrafish. J Fish Biol 2003, 62(4):895-906.

70. Uchida D, Yamashita M, Kitano T, Iguchi T: Oocyte apoptosis during the transition from ovary-like tissue to testes during sex differentiation of juvenile zebrafish. J Exp Biol 2002, 205(Pt 6):711-718.

71. Rodríguez-Marí A, Cañestro C, BreMiller RA, Nguyen-Johnson A, Asakawa K, Kawakami K, Postlethwait JH: Sex reversal in zebrafish fancl mutants is caused by Tp53-mediated germ cell apoptosis. PLoS Genet 2010, 6(7):e1001034

72. Amaral JD, Xavier JM, Steer CJ, Rodrigues CM: The role of p53 in apoptosis. Discov Med 2010, 9(45):145-152.

73. Baeuerle PA, Henkel T: Function and activation of NF-kappaB in the immune system. Annu Rev Immunol 1994, 12(1):141-179.

74. Barnes PJ, Karin M: Nuclear factor-KB - a pivotal transcription factor in chronic inflammatory diseases. N Engl J Med 1997, 336(15):1066-1071.

75. Ghosh S, May MJ, Kopp EB: NF-KB and Rel proteins: evolutionarily conserved mediators of immune responses. Annu Rev Immunol 1998, 16(1):225-260

76. Hong CY, Park JH, Seo KH, Kim JM, Im SY, Lee JW, Choi HS, Lee K: Expression of MIS in the testis is downregulated by tumor necrosis factor alpha through the negative regulation of SF-1 transactivation by NF-kappa B. Mol Cell Biol 2003, 23(17):6000-6012.

77. Pradhan A, Khalaf H, Ochsner SA, Sreenivasan R, Koskinen J, Karlsson M, Karlsson J, McKenna NJ, Orbán L, Olsson P-E: Activation of NF-kB protein prevents the transition from juvenile ovary to testis and promotes ovarian development in zebrafish. J Biol Chem 2012, 287(45):37926-37938.

78. Chen D, Li Z, Yang Q, Zhang J, Zhai Z, Shu H-B: Identification of a nuclear protein that promotes NF-kB activation. Biochem Biophys Res Commun 2003, 310(3):720-724.

79. Pajerowski AG, Nguyen C, Aghajanian H, Shapiro MJ, Shapiro VS: NKAP is a transcriptional repressor of notch signaling and is required for $\mathrm{T}$ cell development. Immunity 2009, 30(5):696-707.

80. Hsiao C-D, Tsai H-J: Transgenic zebrafish with fluorescent germ cell: a useful tool to visualize germ cell proliferation and juvenile hermaphroditism in vivo. Dev Biol 2003, 262(2):313-323.

81. Wang XG, Bartfai R, Sleptsova-Freidrich I, Orban L: The timing and extent of 'juvenile ovary' phase are highly variable during zebrafish testis differentiation. J Fish Biol 2007, 70:33-44.

82. Wu GC: The expression of $a m h$ and $a m h r 2$ is associated with the development of gonadal tissue and sex change in the protandrous black porgy, acanthopagrus schlegeli. Biol Reprod 2010, 83(3):443-453.

83. Wassarman PM: Mammalian fertilization: molecular aspects of gamete adhesion, exocytosis, and fusion. Cell 1999, 96(2):175-183.

84. Hamazaki TS, Nagahama Y, luchi I, Yamagami K: A glycoprotein from the liver constitutes the inner layer of the egg envelope (zona pellucida interna) of the fish, Oryzias latipes. Dev Biol 1989, 133(1):101-110.

85. Cavaco JE, Laurentino SS, Barros A, Sousa M, Socorro S: Estrogen receptors $a$ and $\beta$ in human testis: both isoforms are expressed. Syst Biol Reprod Med 2009, 55(4):137-144.

86. Chakraborty T, Shibata Y, Zhou L-Y, Katsu Y, Iguchi T, Nagahama Y: Differential expression of three estrogen receptor subtype mRNAs in gonads and liver from embryos to adults of the medaka, Oryzias latipes. Mol Cell Endocrinol 2011, 333(1):47-54.
87. Socorro S, Power D, Olsson P, Canario A: Two estrogen receptors expressed in the teleost fish, Sparus aurata: cDNA cloning, characterization and tissue distribution. J Endocrinol 2000, 166(2):293-306.

88. Nelson ER, Habibi HR: Functional significance of nuclear estrogen receptor subtypes in the liver of goldfish. Endocrinology 2010, 151(4):1668-1676.

89. Takahasi H: Juvenile hermaphroditism in the zebrafish, Brachydanio rerio. Bull Fac Fish Hokkaido Univ 1977, 28:57-65.

doi:10.1186/1477-7827-12-5

Cite this article as: Ravi et al: Small-scale transcriptomics reveals differences among gonadal stages in Asian seabass (Lates calcarifer). Reproductive Biology and Endocrinology 2014 12:5.

\section{Submit your next manuscript to BioMed Central and take full advantage of:}

- Convenient online submission

- Thorough peer review

- No space constraints or color figure charges

- Immediate publication on acceptance

- Inclusion in PubMed, CAS, Scopus and Google Scholar

- Research which is freely available for redistribution

Submit your manuscript at www.biomedcentral.com/submit
Biomed Central 